\title{
Prediction of underground cavity roof collapse using the Hoek-Brown failure criterion
}

\author{
A.M. Suchowerska, R.S. Merifield \& J.P. Carter \\ The University of Newcastle, Newcastle, Australia
}

\begin{abstract}
Preventing roof collapse in underground tunnels, natural cavities or mines is a challenge to geotechnical engineering. To evaluate the roof stability of underground cavities two theoretical methods have been used in this study. The rock mass strength has been described by the Hoek-Brown failure criterion. The results of both methods were found to be consistent and have been presented as stability charts for a range of rock properties and geometries. The results of the analysis allow for prediction of roof collapse, but they can also be used to determine whether the failure surface that develops in the rock mass is local or extends through the full depth of cover. This is of significant concern if there are overlying cavities and if estimating surface subsidence.
\end{abstract}

\section{INTRODUCTION}

Underground cavities are frequently constructed for roadway or railway tunnels, for power caverns and in mining. The demand for underground openings is increasing, resulting in cavities needing to be built in close proximity to each other or close to the ground surface. In such cases roof caving or interburden collapse is of primary concern to designers. Three common methods to analyse the stability of underground cavities in rock are assessments made using empirical data, calculations using analytical methods and simulations using numerical modelling.

Empirical data for underground cavity stability has typically been presented in the form of stability charts (Lang 1994, Ouchi et al. 2004). Empirical data for multiple seam coal mining has also been compiled in stability charts (Mark et al. 2007). Both sources of empirical data exhibit large scatter, limiting the accuracy of correlations and therefore the reliability of predictions. Although analytical methods using upper bound (UB) limit analysis have been derived (Lippman 1971, Fraldi \& Guarracino 2009), the equations obtained have not been rigorously investigated. Numerical modelling is widely used in industry as a means of assessing cavity stability, however this method is very time consuming and requires a large number of input variables, which, depending on available data, may need to be estimated or assumed.

In this study the analytical method of Fraldi \& Guarracino (2009) was used to determine stability charts for cavities in rock. To validate further these stability charts, a finite element (FE) upper and lower bound (UB-LB) formulation has also been used. The benefit of deriving such stability charts is that underground cavity designers will have more reliable and easily accessible data to predict the limits for roof collapse.

\section{THEORY}

Underground cavities have been constructed for many: centuries. However, working underground poses. many potential hazards, e.g., roof collapse, gas explosions, gas poisoning and suffocation. The demand to build cavities in close proximity, in parallel with more stringent occupational safety regulations, has been driving the need to understand the variables and parameters governing the stability of underground cavities.

\subsection{Methods to Assess Stability}

\subsubsection{Empirical Data}

In the middle of the 20th century the means of understanding why cavities fail was usually assessed using empirical data. The compiled empirical data was used to develop tunnel support design systems based on a rock mass classification system, viz., the Rock Mass Rating (RMR) (published by Bieniawski 1973) and the $Q$ index (introduced by Barton et al. 1974). Lang (1994) presented a detailed empirical stability chart relating the Rock Mass Rating (RMR) to cavity span (Fig. 1). The stability chart was subdivided into three categories of possible outcomes: stable, possibly unstable and unstable. The chart was then further refined by Ouchi et al. (2004) for cavities in lower quality materials. The uncertainties in the stability zonings are high because of the large distribution in data values.

The significant drawback in using methods based on empirical data is that stability is correlated with 
Modified group classification lines

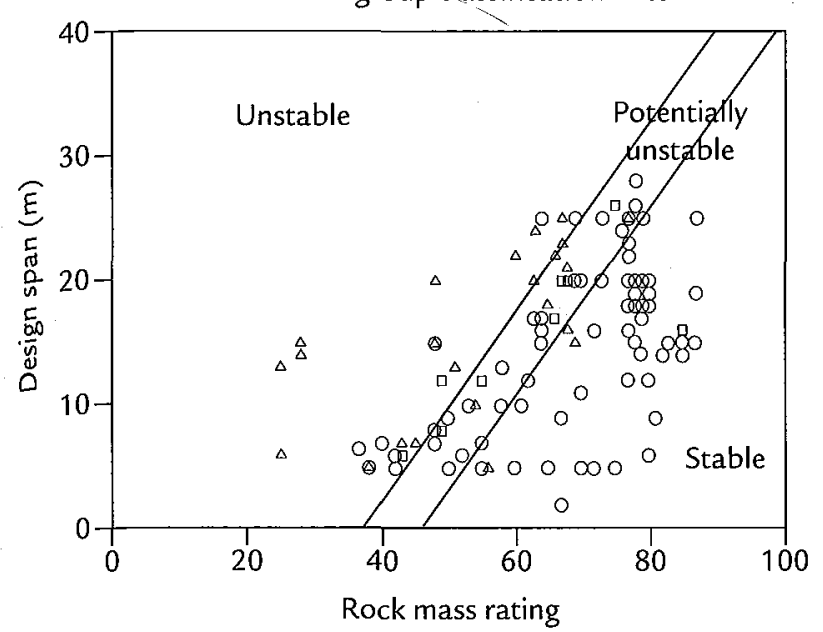

Figure 1 Stability chart of underground cavities presented by Lang (1994).

rock mass classification coefficients RMR or Q. Both the RMR and $Q$ systems are used directly for tunnel support design, and do not give detailed information about the inherent rock mass properties. On the other hand, numerical and analytical methods require specification of the rock mass properties as input variables. For these reasons, it is not generally possible to compare or validate results obtained from numerical or analytical methods with empirical data.

\subsubsection{Numerical Modelling}

The increase in power and accessibility of computers has enabled numerical modelling to become a popular means of investigating underground stability. A large number of variables is required in order to conduct a numerical analysis. These need to be determined either through laboratory or in-situ testing or otherwise assumed. The benefit of this approach, over all other methods, is that it allows for many facets of the failure process to be explored. Variables that govern failure can be identified and the relationships between stabilising and de-stabilising variables can be determined.

\section{2:1.3 Analytical Methods}

Using analytical methods to assess ground stability allows engineers to develop mathematical models to Inderstand the mechanics of failure. Such methods include the Limit Equilibrium Method (LEM) and Limit Analysis (LA) based on plasticity theory. These methods are widely used in practice due to their simplicity and generality, yet they are usually underpinned by significant simplifying assumptions.

Limit analysis is a very powerful means of conducting analytical analysis. Limit analysis involves enveloping the theoretical solution by determining UB-LB for the true collapse load. The true collapse oad can be approximated well if the difference betheen the UB and LB is small.

\subsection{Failure Criterion}

A geotechnical stability analysis requires a failure criterion to be assigned for the materials involved in the problem being assessed. The Mohr-Coulomb failure criterion has been widely used in geotechnical engineering stability problems for many decades. For this case failure is governed by the cohesion and friction angle of the material as well as the normal forces on the failure plane (Equation 1). Its popularity and easy use in many applications has lead to it being the primary failure criterion offered by numerical analysis packages.

$\tau=c+\sigma_{\mathrm{n}} \tan \varphi$

An alternative empirical failure criterion for rock masses was developed by Hoek \& Brown (1997), which essentially involved the fitting of curves to the results of triaxial tests on rock samples (Equation 2). An updated version of the original criterion refined the effects observed in lower strength materials (Hoek et al. 2002). This criterion requires the materials to be classified using the Geological Strength Index (GSI), and assumes that the rock mass behaves essentially as an isotropic material. Such conditions are relevant for either an intact rock or otherwise a heavily jointed rock with randomly oriented discontinuities. However, this failure criterion does not apply if there is a preferential failure mechanism as a result of a set of parallel discontinuities.

$\sigma_{1}^{\prime}=\sigma_{3}^{\prime}+\sigma_{\mathrm{ci}}\left(m_{\mathrm{b}} \frac{\sigma_{3}^{\prime}}{\sigma_{\mathrm{ci}}}+s\right)^{\mathrm{a}}$

Each of the Mohr-Coulomb and Hoek-Brown failure criteria has its place in geotechnical engineering analysis. The Mohr-Coulomb failure criterion permits evaluation of the maximum shear stress on a failure surface as simply the sum of the cohesion and the combination of friction angle and normal stress on the failure plane. In contrast, the Hoek-Brown failure criterion indicates that the maximum principal stress $\left(\sigma_{1}\right)$ has a non-linear relationship with the minimum confining stress $\left(\sigma_{3}\right)$. When both failure criteria are plotted as maximum vs. minimum principal stresses, the Mohr-Coulomb failure criterion usually indicates a larger principal stress capacity than the Hoek-Brown criterion at both low and high confining stresses (Hoek et al. 2002).

\subsection{Limit Analysis of Underground Cavities}

Upper bound limit analysis of underground cavities has been considered using both the Mohr-Coulomb and Hoek-Brown failure criteria. Lippmann (1971) presented the results of limit analysis for an underground cavity considering an UB mechanism using a Mohr-Coulomb failure criterion and its associated flow rule. The cavity was assumed to be rectangular. This UB solution was developed enabling the adoption 
of either the Mohr-Coulomb or Hoek-Brown failure criterion (Fraldi \& Guarracino 2009). For cases in which it assumed that the rock mass fails according to the Hoek-Brown criterion, application of this method involves fitting Equation 3 to the HoekBrown criterion by calculating appropriate values for the dimensionless parameters $A$ and $B$. These parameters are defined in the following equation, which is no more than a re-expression of the HoekBrown failure criterion in terms of shear and normal stress components acting on the failure surface, i.e.,

$\tau= \pm A \sigma_{\mathrm{ci}}\left[\frac{\left(\sigma_{\mathrm{n}}+\sigma_{\mathrm{t}}\right)}{\sigma_{\mathrm{ci}}}\right]^{\mathrm{B}}$

Values of $A$ and $B$ can be çalculated by considering the equivalence of the two different forms of expression of the Hoek-Brown criterion, i.e., Equations (2) and (3), one expressed in terms of principals stresses (Equation 2) and the other expressed in terms of normal and shear stresses acting on the failure surface (Equation 3). Exact closed-form expressions for $A$ and $B$ cannot be established for the general case, so that values of the parameters $A$ and $B$ are normally determined numerically using the regression method presented in Hoek (2000), where eight increments are used for values of $\sigma_{3}$ between 0 to $25 \%$ $\sigma_{\mathrm{ci}}$. This recommended method has been adopted for this study. It is noted that Equation 3 represents the Mohr-Coulomb failure criterion exactly for the special case where $A=\tan \phi, B=1$, and $\sigma_{\mathrm{t}}=c(\tan \phi)^{-1}$.

\subsection{Finite Element Upper and Lower Bound Formulation}

The FE UB-LB formulations developed by Lyamin $\&$ Sloan (2002a, b) and Krabbenhoft et al. (2005) generally yield more accurate results than analytical limit analysis predictions determined by hand calculations. The FE formulations involve iterative calculations which aim to refine the margin between the two bound values. Further, the mesh used in the computer analysis may include discontinuities, which can therefore allow for abrupt changes in stresses in the lower bound formulation and in the velocities in the upper bound formulation.

\section{PROBLEM DEFINITION}

In order to assess cavity stability a plane strain model was developed, as shown in Figure 2. Details of the values of the variables assumed in the analysis are presented in Table 1. The model was designed to represent a cavity in a rock mass that is surrounded by a uniform material. This is an idealised representation of either a multiple seam mine where there is no extra stresses due to goaf loading on the overlying seam, or a tunnel at shallow depth where there is

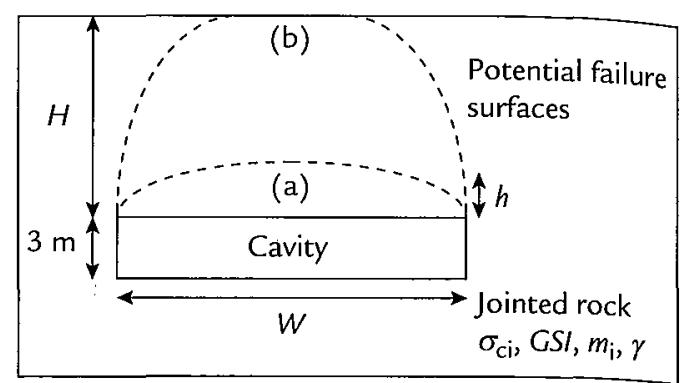

Figure 2 Schematic representation of the problem analysed in this study.

Table 1 Variables considered in the analysis

\begin{tabular}{lll}
\hline Variable & Description & Values considered \\
\hline$G S I$ & Material property & 10 to 90 , inc of 10 \\
$m_{\mathrm{i}}$ & Material property & 5 to 30, inc of 5 \\
$H$ & Cover depth & 10 to 80, inc of 10 \\
$W$ & Width of rectangular cavity & 10 to 80, inc of 10 \\
$\gamma$ & Unit weight of material & Optimised \\
$\sigma_{\mathrm{ci}}$ & Compression strength of & $100 \mathrm{MPa}$ \\
& intact rock & \\
\hline
\end{tabular}

a uniform overlying material. The term 'cover depth' will be used to represent either the interburden depth in multiple seam situations or the depth of cover in a shallow underground cavity.

\section{SOLUTION OF THE PROBLEM}

Two methods were considered in this study to solve this problem. The first is an analytical closed form UB analysis (Fraldi \& Guarracino 2009). The second is a numerical finite element UB-LB formulation (Lyamin \& Sloan 2002a, b, Krabbenhoft et al. 2005). Two analysis methods were used to increase the reliability of the numerical results.

\subsection{Closed Form Upper Bound Analysis (CF-UB)}

Upper bound equations presented by Fraldi \& Guarracino (2009) indicate that the critical halfwidth of an excavation $(L)$ and maximum height of the collapsing block $(b)$ can be calculated by Equations (4) and (5) respectively. The critical unit weight that would cause the roof to collapse was calculated by doubling the half-width (since $W=2 L$ ), equating it to the desired span and optimising for the density.

$L=A B^{-\mathrm{B}}(1+B)^{\mathrm{B}}(\gamma)^{-1} \sigma_{\mathrm{c}}^{(1-\mathrm{B})} \sigma_{\mathrm{r}}^{\mathrm{B}}$

$b=\frac{(1+B) \sigma_{\mathrm{t}}}{\gamma B}$

This upper bound derivation assumes that the cavity was at sufficient depth that the thickness of the collapsing block would never reach the surface (i.e., $H>b$ ). In the model considered here at large cavity spans, relative to cover depth, this assumption is not 
always true. The corresponding span $(W / H)$ when the collapsing block height equals the cover depth can be calculated by substituting the cover depth $(H)$ into Equation (5) and rearranging for $\gamma$ (Equation 6). Substituting Equation (6) and $2 L=W$ into Equation (4), provides the critical half width for a collapsing block penetrating through the cover depth, as Equation (7).

$\gamma=\frac{(1+B) \sigma_{\mathrm{t}}}{H B}$

$\frac{W}{H}=\frac{2 A B^{-\mathrm{B}}(1+B)^{\mathrm{B}} \sigma_{c}^{(1-\mathrm{B})} \sigma_{\mathfrak{t}}^{\mathrm{B}} B}{(1+B) \sigma_{\mathrm{t}}}$

\subsection{Finite Element Formulation}

The FE formulation required the model to be built using a computer script. The material properties were kept constant throughout the section profile, with two rough interfaces placed at the top and the bottom of the cavity pillar (Fig. 3). The formulation then meshed the section based upon the minimum number of elements prescribed in the script. The number of elements was made large enough to ensure a maximum margin of $10 \%$ between the LB and UB results.

\section{RESULTS}

Dimensionless stability parameters $N$ and $M$ are used to present the results and are defined in Equation (8).

$N=\frac{\sigma_{\mathrm{ci}}}{\gamma W F}, \quad M=\frac{\sigma_{\mathrm{ci}}}{\gamma H F}$

where $F$ is the factor of safety against failure.

\subsection{Closed Form Upper Bound Analysis}

Figure 4 shows the results obtained from calculations using the method proposed by Fraldi \& Guarracino

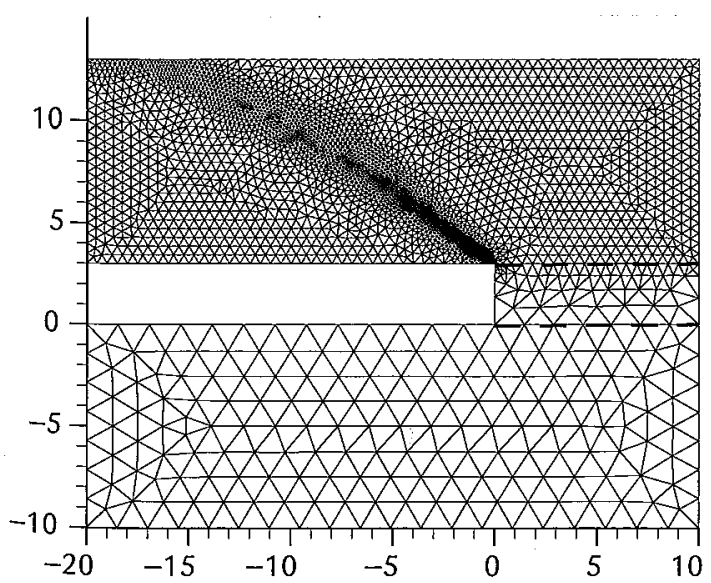

Figure 3 Model used in the FE Limit Analysis method. rough Interfaces (dashed lines) were placed at top and bottom of pillars. Dimensions are given in metres.
(2009), for $m_{\mathrm{i}}$ values of 20 . The results indicate that lower GSI values required larger stability parameters ( $N$ or $M)$ than higher GSI values, in order to be stable.

The dashed lines in both charts in Figure 4 show the limit when the rigid collapsing block extended through the whole cover depth (i.e., when $H=b$ ). This was determined using the method outlined in Section 4. The maximum achievable span for a given cover depth is observed to occur for a value of GSI of 20 to 30 .

The results corresponding to all other values of $m_{\mathrm{i}}$ considered in the study can be scaled off the values obtained for $m_{\mathrm{i}}$ of 20 . The stability factors obtained from Figure 4, can be scaled to account for other values of $m_{\mathrm{i}}$ using Figure 5.

\subsection{Finite Element Upper and Lower Bounds}

The results from the FE formulation are an upper and lower bound to the true collapse load. They have been solved to within a $10 \%$ margin of each other. The results are presented in terms of the stability factors $N$ in Figure 6.

There is a common trend between the stability factor $N$ and the ratio $W / H$, which is observed over
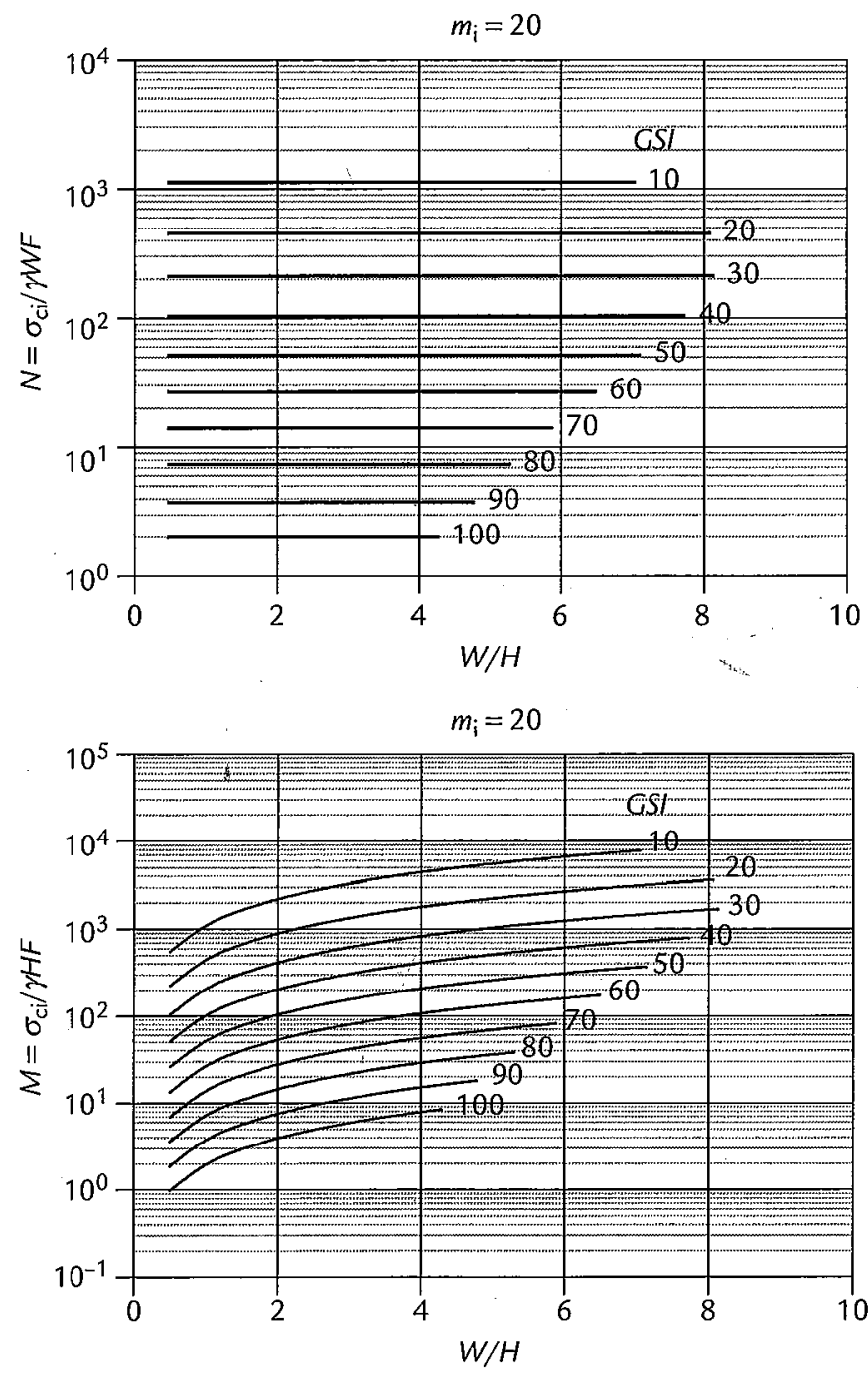

Figure 4 Stability charts for $m_{i}$ of 20 , obtained using the CF-UB analysis. 


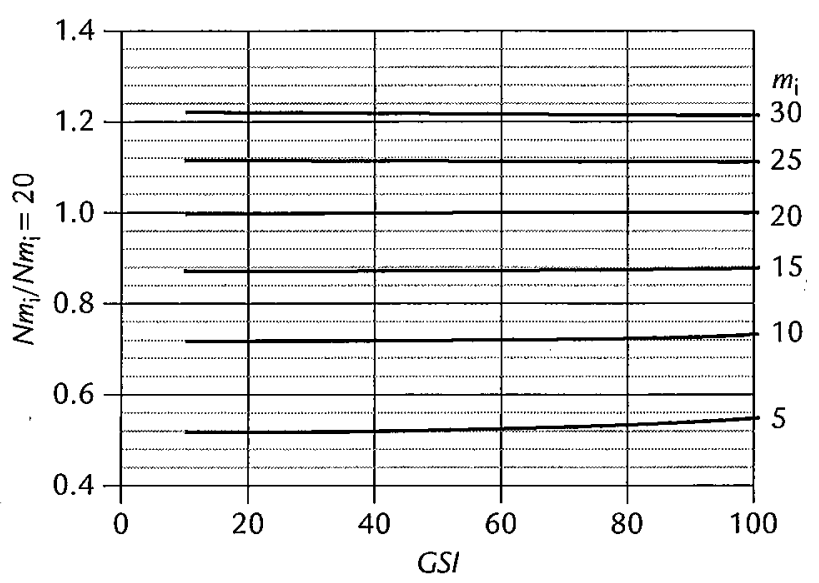

Figure 5 Scaling for the stability factors ( $N$ and $M$ ), obtained from Figure 4, for $m_{i}$ values other than 20 .

all the values of $m_{\mathrm{i}}$. The stability factor $N$ is constant at low values of $W / H$. This coincides with the collapsed surface not extending through the entire height of the cover depth $(H)$. At higher values of $W / H$ the value of $N$ increases, and this corresponds to the failure surface extending through the full cover depth. These two failure surfaces are shown schematically in Figure 2 as curves (a) and (b) respectively. The change in slope in these plots indicates the boundary between the two cases where (a) the failure surface does not extend through the full cover depth, and (b) the failure surface extends through the full cover depth. As an example, this point is labelled $Z$ for $m_{\mathrm{i}}$ of 20 and GSI of 90 in Figure 6. Charts of stability factor $N$ for the values of $m_{\mathrm{i}}$ considered here, were obtained using the UB (solid line) and LB (dashed line) formulations.

\section{DISCUSSION}

Figure 7 shows the stability factor $N$ obtained from both methods considered in this study for a value of $m_{\mathrm{i}}$ of 20 . The results of the CF-UB analysis lie between the FE UB and LB formulation results.

The CF-UB analysis appears to overestimate the ratio $W / H$ when the maximum height of the collapsing block $(b)$ equals the cover depth $(H)$. There are two notable reasons why this has occurred. Firstly, the calculation derived in Section 4 assumes that the remaining strata left behind, after the roof has collapsed, will be stable. When the depth of the collapsing block $(b)$ approaches the full depth of cover $(H)$, this becomes very unlikely in practice. The CF-UB analysis does not assess the stability of the remaining strata, while the FE UB-LB method does.

The other notable reason why there is a difference between the two methods is because of the regression method used to calculate suitable values of the parameters $A$ and $B$ in Equation (3). It is necessary to curve fit Equation (3) to the true shear strength envelope in order to use the equations derived by Fraldi $\&$ Guarracino (2009). The regression undertaken in
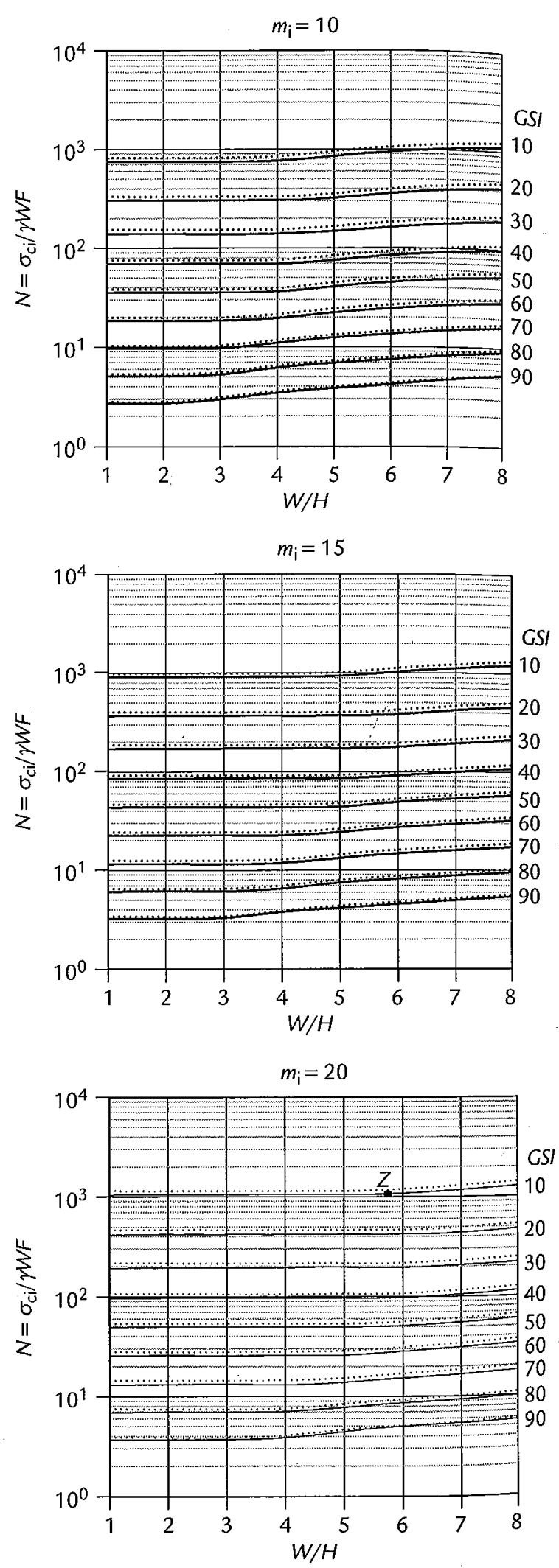

Figure 6 Charts of stability factor $N$ obtained using the UB (solid line) and LB (dashed line) formulations.

this study used the recommendation by Hoek (2000), where 8 increments were used over a range of 0 to $25 \%$ of $\sigma_{\mathrm{ci}}$. Experience has shown that varying the range over which the regression is conducted and the number of increments used, can significantly change the values of $A$ and $B$, and therefore the final results of the limit analysis. This issue will be investigated further in a subsequent paper. 


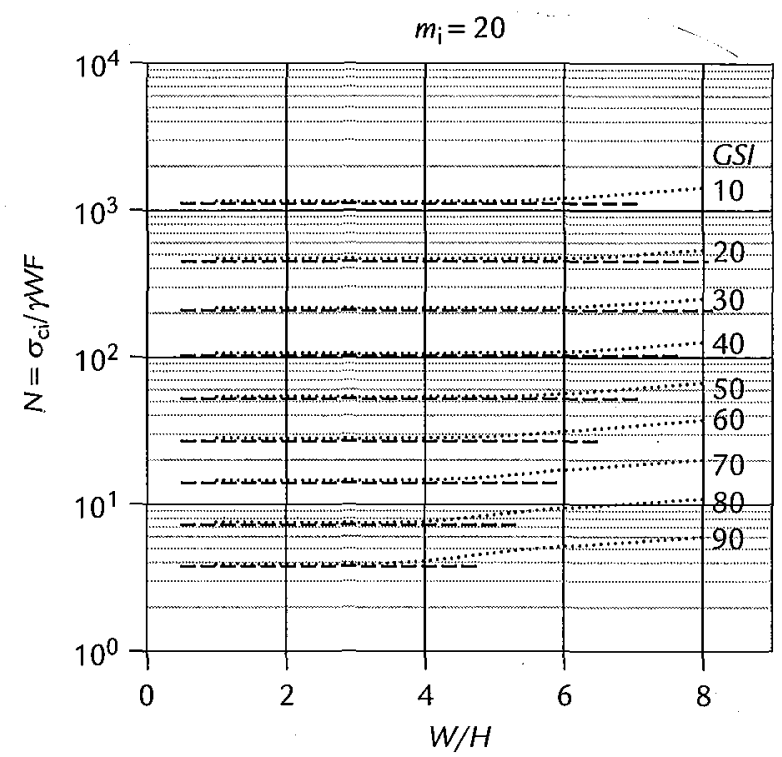

Figure 7 Stability charts $m_{\mathrm{i}}=20$ with results from CF-UB (dashed line) and FE-LB (dotted line) formulations.

\subsection{Application Example}

The charts presented in Figures 4-6 can be used to calculate the factor of safety for a proposed cavity. The surrounding rock mass needs to be assessed according to GSI and the geometry of the proposed cavity and the cover depth need to be known. As an example, a $40 \mathrm{~m}$ wide cavity is to be constructed in medium strength sandstone $\left(\sigma_{\mathrm{ci}}=80 \mathrm{MPa}\right)$, with a unit weight of $20 \mathrm{kN} / \mathrm{m}^{3}$. The GSI and $m_{\mathrm{i}}$ have been quantified as 40 and 20 respectively. The interburden depth to an overlying cavity is $30 \mathrm{~m}$. Therefore $W / H=1.34$, and thus Figure 6 indicates $N \sim 90$. The factor of safety can then be calculated as $F=\sigma_{\text {cil }}$ / $\gamma W N=80000 / 20 * 40 * 90=1.11$.

This scenario can also be assessed by determining the maximum width cavity for a given factor of safety. If a minimum factor of safety of 2 is required the dimensionless parameter $M$ is calculated: $M=\sigma_{\mathrm{ci}} /$ $\gamma H F=80000 / 20 * 30 * 2=66.67$. Using Figure 4, for $G S I=40$, a maximum value of $W / H$ is approximately 0.6 . Therefore the maximum cavity width would be $0.6^{*} 30=18 \mathrm{~m}$

\section{CONCLUSION}

In this study we have presented stability charts for underground cavities using the Hoek-Brown failure criterion. The two methods of analysis yielded results that generally agree well with each other.

The proposed stability charts enable designers of underground cavities to predict the safe widths of underground cavities more quickly. They also show whether the failure surface will be localized or otherwise extend through the whole cover depth.

\section{REFERENCES}

Barton N.R, Lien R. \& Lunde J. 1974. Engineering classification of rock masses for design of tunnel support, Rock Mechanics 6(4):189-239.

Bienawski Z.T., 1973. Engineering classification of jointed rock masses, Trans South African Institute of Civil Engineers. 15:335-344.

Chen W.F., 1975. Limit Analysis and Soil Plasticity. Amsterdam: Elsevier Scientific.

Fraldi M. \& Guarracino F. 2009. Limit analysis of collapse mechanisms in cavities and tunnels according to the Hoek-Brown failure criterion, International Journal of Rock Mechanics and Mining Science 46:665-673.

Hoek E. \& Brown E.T. 1997. Practical estimates of rock mass strength, International Journal of Rock Mechanics and Mining Science, 34(8):1165-1186.

Hoek E., Carranza-Torres C. \& Corkum B. 2002. Hoek-Brown failure criterion-2002 Edition, 161-201, Rotterdam: Balkema.

Hoek E. 2000. Rock Engineering-Course notes by Evert Hoek, Proceedings of the North American Rock Mechanics Society, meeting in Toronto.

Krabbenhoft K., Lyamin A.V., Hjiaj M. \& Sloan S.W. 2005. A new discontinuous upper bound limit analysis formulation, International Journal for Numerical Methods in Engineering, 63:1069-1088.

Lang B.D.A. 1994. Span design for entry-type excavations.

Lippman H. 1971. Plasticity in Rock Mechanics, International Journal of Mechanical Sciences 13:291-297.

Lyamin A.V. \& Sloan S.W. 2002a. Lower bound limit analysis using non-linear programming, International Journal for Numerical and Analytical methods in Geomechanics, 26(2): $181-216$.

Lyamin A.V. \& Sloan S.W. 2002b. Upper bound limit analysis using the linear finite element and non-linear programming, International Journal for Numerical methods in Engineering 55(5):573-611.

Mark C., Chase F. \& Pappas D.M. 2007. Analysis of multiple seam stability, Proceedings of the 26th International Conference on Ground Control in Mining, 5-18

Ouchi A.M, Pakalnis R. \& Brady T.M. 2004. Update of span design curve for weak rock masses, Presented at 2004 $A G M-C I M$ Edmonton. 